EPJ Web of Conferences 37, 08006 (2012)

DOI: $10.1051 /$ epjconf/20123708006

(C) Owned by the authors, published by EDP Sciences, 2012

\title{
Phenomenology of light mesons within a chiral approach
}

\author{
F. Giacosa $^{1}$, D. Parganlija ${ }^{1,2}$, P. Kovács ${ }^{2,3}$, and Gy. Wolf ${ }^{3}$
}

${ }^{1}$ Institute for Theoretical Physics, Johann Wolfgang Goethe University, Max-von-Laue-Str. 1, D-60438 Frankfurt am Main, Germany

${ }^{2}$ Institute for Theoretical Physics, Vienna University of Technology, Wiedner Hauptstr. 8-10, A-1040 Vienna, Austria

${ }^{3}$ Institute for Particle and Nuclear Physics, Wigner Research Center for Physics, Hungarian Academy of Sciences, H-1525 Budapest, Hungary

\begin{abstract}
The so-called extended linear sigma model is a chiral model with (pseudo)scalar and (axial-)vector mesons. It is based on the requirements of (global) chiral symmetry and dilatation invariance. The purpose of this model is the description of the hadron phenomenology up to $1.7 \mathrm{GeV}$. We present the latest theoretical results, which show a good agreement with the experiment.
\end{abstract}

In this paper we describe a chiral $\sigma$ model, called 'extended Linear $\sigma$ model (El $\sigma \mathrm{m})$ ', in which scalar, pseudoscalar, vector, axial-vector quark-antiquark mesons and, in addition, a scalar dilaton/glueball field are the basic degrees of freedom. The aim is to develop a model with the basic symmetries of QCD which can describe the vacuum phenomenology up to $1.7 \mathrm{GeV}$. The Lagrangian of the model is built by requiring (i) global chiral symmetry and (ii) dilatation invariance. Although chiral models are studied since long time [1], the here presented El $\sigma \mathrm{m}$ represents the first attempt to treat in a unified chiral framework (pseudo)scalar mesons (including the glueball) as well as (axial-)vector ones. (Previous studies [2] exist only for $N_{f}=2$ and not all the mentioned d.o.f. were taken into account.) It turns out that the inclusion of (axial-)vector d.o.f. have a very strong influence on the overall phenomenology, influencing also the decays in the (pseudo)scalar sector.

The explicit form of the Lagrangian in the mesonic sector reads (for a generic number of flavors $\left.N_{f}\right)$ [3-6]:

$$
\begin{gathered}
\mathcal{L}_{E l \sigma m}=\frac{1}{2}\left(\partial_{\mu} G\right)^{2}-V_{d i l}(G)+\operatorname{Tr}\left[\left(D^{\mu} \Phi\right)^{\dagger}\left(D_{\mu} \Phi\right)-a G^{2} \Phi^{\dagger} \Phi-\lambda_{2}\left(\Phi^{\dagger} \Phi\right)^{2}\right]-\lambda_{1}\left(\operatorname{Tr}\left[\Phi^{\dagger} \Phi\right]\right)^{2}+ \\
+c_{1}\left(\operatorname{det} \Phi^{\dagger}-\operatorname{det} \Phi\right)^{2}+\operatorname{Tr}\left[H\left(\Phi^{\dagger}+\Phi\right)\right]-\frac{1}{4} \operatorname{Tr}\left[\left(L^{\mu v}\right)^{2}+\left(R^{\mu v}\right)^{2}\right] \\
+\frac{b}{2} G^{2} \operatorname{Tr}\left[\left(L^{\mu}\right)^{2}+\left(R^{\mu}\right)^{2}\right]+\frac{1}{2} \operatorname{Tr}\left[\hat{\delta}\left(L^{\mu}\right)^{2}+\left(R^{\mu}\right)^{2}\right]-2 i g_{2}\left(\operatorname{Tr}\left[L_{\mu \nu}\left[L^{\mu}, L^{v}\right]\right]+\operatorname{Tr}\left[R_{\mu \nu}\left[R^{\mu}, R^{v}\right]\right]\right) \\
+\frac{h_{1}}{2} \operatorname{Tr}\left[\left(\Phi^{\dagger} \Phi\right)\left(L_{\mu}^{2}+R_{\mu}^{2}\right)\right]+h_{2} \operatorname{Tr}\left[\Phi^{\dagger} L_{\mu} L^{\mu} \Phi+\Phi R_{\mu} R^{\mu} \Phi\right]+2 h_{3} \operatorname{Tr}\left[\Phi R_{\mu} \Phi^{\dagger} L^{\mu}\right]+\ldots
\end{gathered}
$$

where $D^{\mu} \Phi=\partial^{\mu} \Phi-i g_{1}\left(L^{\mu} \Phi-\Phi R^{\mu}\right)$ and dots represent further terms which are unimportant in the evaluation of decays and (on-shell) scattering lengths. Following comments are in order:

(i) The (pseudo)scalar quark-antiquark mesons are described by the matrix $\Phi=\left(S^{a}+i P^{a}\right) t^{a}\left(t^{a}\right.$ are the generators of the group $U\left(N_{f}\right)$ ). The pseudoscalar states are the pion, kaon and the $\eta$ and $\eta^{\prime}$ mesons. The assignment of scalar states is controversial [6-8] and represents one of the motivations of our study. It turns out that the best agreement with the experiment is obtained when the quarkantiquark scalar states of the model are assigned to the scalar resonances between 1-2 GeV (theory in Refs. [3-5] and experimental results in Ref. [9]).

This is an Open Access article distributed under the terms of the Creative Commons Attribution License 2.0, which permits unrestricted use, distribution, and reproduction in any medium, provided the original work is properly cited. 
(ii) The (axial-)vector mesons are described by the matrices $L^{\mu}=\left(V^{a, \mu}+A^{a, \mu}\right) t^{a}$ and $R^{\mu}=\left(V^{a, \mu}-\right.$ $\left.A^{a, \mu}\right) t^{a}$. The vector mesons are the usual $\rho, \omega, K^{*}(892)$, and $\phi$ mesons. The axial-vector mesons are assigned to $a_{1}(1230), K_{1}(1270), f_{1}(1285)$ and $f_{1}(1510)$.

(iii) The dilaton field is denoted by $G$ and its potential reads $V_{d i l}(G)=\frac{1}{4} \frac{m_{G}^{2}}{\Lambda_{G}^{2}}\left[G^{4} \ln \left(\frac{G}{\Lambda_{G}}\right)-\frac{G^{4}}{4}\right][10]$. The parameter $\Lambda_{G} \sim N_{c} \Lambda_{Q C D}$ sets the energy scale of the theory.

(iv) The $U(1)_{A}$ anomaly term is parametrized by the parameter $c_{1}$, which has dimension [Energy ${ }^{4-2 N_{f}}$.

(v) The matrix $H \propto \operatorname{diag}\left\{m_{u}, m_{d}, m_{s}, \ldots\right\}$ describes explicit symmetry breaking of both chiral and dilatation symmetries due to the bare quark masses $m_{i}$. Similarly, in the (axial-)vector sector the diagonal matrix $\hat{\delta}$ has been introduced.

(vi) Chiral symmetry breaking takes place when the parameter $a$ is negative. In fact, upon the condensation of the field $G=G_{0}$, the 'wrong' sign for mesonic masses $a G_{0}^{2}<0$ sign is realized.

Once the shifts of the scalar fields $G \rightarrow G_{0}+G$ and $\Phi \rightarrow \operatorname{diag}\left\{\sqrt{2} \sigma_{N}, \sqrt{2} \sigma_{N}, \ldots\right\}+\Phi$, where the first term is a diagonal matrix with the quark-antiquark condensates, and necessary redefinitions of the pseudoscalar and axial-vector fields have been performed [3,5], the explicit calculations of physical processes are lengthy but straightforward. (Note, the calculations are performed at tree-level; the inclusion of loops is a task for the future, but only slight changes are expected [11].)

In the following we summarize the results that we have obtained with the model in Eq. (1):

- $N_{f}=2$ with frozen glueball $\left(m_{G} \rightarrow \infty\right)$ [3,12]: by considering the limit $m_{G} \rightarrow \infty$ the dilaton/glueball field is not an active d.o.f. One can neglect the gluonic part of the Lagrangian of Eq. (1) and set $G=G_{0}=\Lambda_{G}$. In the works in Refs. [3,12] it has been shown that the inclusion of (axial-)vector mesons has a strong influence on the overall phenomenology. For instance, the width of the scalar meson $\sigma$ (the chiral partner of the pion) decreases substantially w.r.t. the case without (axial-)vector mesons: for this reason, the identification of this field with the resonance $f_{0}(500)$ is not favoured, because the theoretically evaluated width is smaller than $200 \mathrm{MeV}$; this is at odd with the experiment, according to which it is larger than $400 \mathrm{MeV}$. On the other hand, the identification of the $\sigma$ field with the resonance $f_{0}(1370)$ turns out to be in agreement with the experimental results. The description of the (axial-)vector resonances is also in agreement with the experiments reported in Ref. [9].

- $N_{f}=2$ with active glueball $\left(m_{G} \sim 1.5 \mathrm{GeV}\right)$ [4]: the glueball with a bare mass $m_{G} \sim 1.5 \mathrm{GeV}$, in agreement with the lattice results [13], has been investigated for the first time in a chiral model with (axial-)vector mesons. (For other approaches see Ref. [8] and refs. therein). The state $f_{0}(1500)$ results as the predominantly $(75 \%)$ glueball state, and the rest of the phenomenology is only slightly affected w.r.t. the previous case, in which $m_{G} \rightarrow \infty$. Moreover, also the gluon condensate has been evaluated and is in agreement with lattice results.

- $N_{f}=3$ with frozen glueball $\left(m_{G} \rightarrow \infty\right)$ [5,14]: the results for this scenario are interesting because, for the first time, it is possible to study in a chiral framework the overall vacuum's phenomenology up to $1.7 \mathrm{GeV}$. The results for the best-fit scenario are listed in Table I. Eleven free parameters enter in the fit and the total $\chi^{2} \simeq 1$ signalizes a good agreement of the theory with the experiment, see Ref. [5] for details. It is visible that the resonances $a_{0}(1450)$ and $K_{0}^{*}(1430)$ are well described as quark-antiquark fields. The scalar-isoscalar mesons are not included in the fit, but can be studied as a consequence of it: the decay pattern and the masses suggest that $f_{0}(1370)$ and $f_{0}(1710)$ are (predominantly) the non-strange and strange scalar-isoscalar fields.

- Other works related to the model: in Ref. [15] the baryonic part of the model has been presented for $N_{f}=2$ and in Ref. [16] the nonzero density and nuclear matter saturation have been described. In Ref. [17] part of the model has been investigated at nonzero temperature.

In the future we plan to perform the following investigations with the chiral model in Eq. (1): (i) The case $N_{f}=3$ with $m_{G} \sim 1.5 \mathrm{GeV}$ : the inclusion of an active glueball represents the most straightforward extension of our approach; the aim is a full description of scalar-isoscalar states between 1-2 $\mathrm{GeV}$, including also the resonance $f_{0}(1500)$ [8]. (ii) The case $N_{f}=4$ (that is, including charmonia states) can be studied. In this way we can test the implications of chiral symmetry also beyond the low-energy sector. Note, only two additional parameters (both connected to the charm mass) w.r.t. the 


\begin{tabular}{|c|c|c|}
\hline Observable & Fit $[\mathrm{MeV}]$ & Experiment $[\mathrm{MeV}]$ \\
\hline$f_{\pi}$ & $96.3 \pm 0.7$ & $92.2 \pm 4.6$ \\
\hline$f_{K}$ & $106.9 \pm 0.6$ & $110.4 \pm 5.5$ \\
\hline$m_{\pi}$ & $141.0 \pm 5.8$ & $137.3 \pm 6.9$ \\
\hline$m_{K}$ & $485.6 \pm 3.0$ & $495.6 \pm 24.8$ \\
\hline$m_{\eta}$ & $509.4 \pm 3.0$ & $547.9 \pm 27.4$ \\
\hline$m_{\eta^{\prime}}$ & $962.5 \pm 5.6$ & $957.8 \pm 47.9$ \\
\hline$m_{\rho}$ & $783.1 \pm 7.0$ & $775.5 \pm 38.8$ \\
\hline$m_{K^{\star}}$ & $885.1 \pm 6.3$ & $893.8 \pm 44.7$ \\
\hline$m_{\phi}$ & $975.1 \pm 6.4$ & $1019.5 \pm 51.0$ \\
\hline$m_{a_{1}}$ & $1186 \pm 6$ & $1230 \pm 62$ \\
\hline$m_{f_{1}(1420)}$ & $1372.5 \pm 5.3$ & $1426.4 \pm 71.3$ \\
\hline$m_{a_{0}}$ & $1363 \pm 1$ & $1474 \pm 74$ \\
\hline$m_{K_{0}^{\star}}$ & $1450 \pm 1$ & $1425 \pm 71$ \\
\hline$\Gamma_{\rho \rightarrow \pi \pi}$ & $160.9 \pm 4.4$ & $149.1 \pm 7.4$ \\
\hline$\Gamma_{K^{\star} \rightarrow K \pi}$ & $44.6 \pm 1.9$ & $46.2 \pm 2.3$ \\
\hline$\Gamma_{\phi \rightarrow \bar{K} K}$ & $3.34 \pm 0.14$ & $3.54 \pm 0.18$ \\
\hline$\Gamma_{a_{1} \rightarrow \rho \pi}$ & $549 \pm 43$ & $425 \pm 175$ \\
\hline$\Gamma_{a_{1} \rightarrow \pi \gamma}$ & $0.66 \pm 0.01$ & $0.64 \pm 0.25$ \\
\hline$\Gamma_{f_{1}(1420) \rightarrow K^{\star} K}$ & $44.6 \pm 39.9$ & $43.9 \pm 2.2$ \\
\hline$\Gamma_{a_{0}}$ & $266 \pm 12$ & $265 \pm 13$ \\
\hline$\Gamma_{K_{0}^{\star} \rightarrow K \pi}$ & $285 \pm 12$ & $270 \pm 80$ \\
\hline
\end{tabular}

Table 1. Best-fit results for masses and decay widths compared with experiment.

case $N_{f}=3$ are needed. The Lagrangian is still the one in Eq. (1). (iii) $\tau$ decays involving (axial-)vector mesons: after including the weak-gauge bosons $W^{ \pm}, Z^{0}$ in the model the spectral functions of vector and axial-vector states can be investigated. (iv) Scalar states below $1 \mathrm{GeV}$ : these states are not part of the model, but could be added as tetraquarks along the line of Ref. [18] (see also Ref. [19] and refs. therein). (v) Inclusion of the pseudoscalar glueball and the evaluation of its decays: this project can be relevant for future experiments, suched as the PANDA experiment at FAIR. (vi) In-medium properties and the phase diagram: the study of chiral phase transition represents an important outlook of this work (for preliminary works see Refs. $[16,17]$ ). In fact, a great advantage of the linear realization of chiral symmetry is the straightforward extension to nonzero temperature and density. Moreover, also inhomogeneous condensates can be investigated.

Acknowledgments Gy. Wolf and P. Kovacs thank the Goethe University for hospitality. They were partially supported by the Hungarian OTKA funds T71989 and T101438. The work of D. Parganlija and F. Giacosa was supported by the Foundation of the Polytechnical Society Frankfurt. This work was financially supported by the Helmholtz International Center for FAIR within the framework of the LOEWE program (Landesoffensive zur Entwicklung Wisschenschaftlich-Ökonomischer Exzellenz) launched by the State of Hesse.

\section{References}

1. S. Gasiorowicz and D. A. Geffen, Rev. Mod. Phys. 41, 531 (1969).

2. P. Ko and S. Rudaz, Phys. Rev. D 50, 6877 (1994). M. Urban, M. Buballa, J. Wambach, Nucl. Phys. A697 (2002) 338-371.

3. D. Parganlija, F. Giacosa, D. H. Rischke, Phys. Rev. D82 (2010) 054024.

4. S. Janowski, D. Parganlija, F. Giacosa, D. H. Rischke, Phys. Rev. D84 (2011) 054007.

5. D. Parganlija, P. Kovacs, G. Wolf, F. Giacosa and D. H. Rischke, arXiv:1208.0585 [hep-ph].

6. F. Giacosa, Phys. Rev. D80 (2009) 074028. F. Giacosa, AIP Conf. Proc. 1322 (2010) 223-231. 
7. C. Amsler and N. A. Tornqvist, Phys. Rept. 389, 61 (2004). E. Klempt and A. Zaitsev, Phys. Rept. 454 (2007) 1

8. C. Amsler and F. E. Close, Phys. Rev. D 53 (1996) 295; W. J. Lee and D. Weingarten, Phys. Rev. D 61, 014015 (2000); F. E. Close and A. Kirk, Eur. Phys. J. C 21, 531 (2001); F. Giacosa, T. Gutsche, V. E. Lyubovitskij and A. Faessler, Phys. Rev. D 72, 094006 (2005); F. Giacosa, T. Gutsche, V. E. Lyubovitskij and A. Faessler, Phys. Lett. B 622, 277 (2005); F. Giacosa, T. Gutsche and A. Faessler, Phys. Rev. C 71, 025202 (2005); H. Y. Cheng, C. K. Chua and K. F. Liu, Phys. Rev. D 74 (2006) 094005; L. Bonanno and A. Drago, Phys. Rev. C 79, 045801 (2009); V. Mathieu, N. Kochelev and V. Vento, Int. J. Mod. Phys. E 18 (2009) 1.

9. K. Nakamura et al. (Particle Data Group), J. Phys. G 37, 075021 (2010).

10. A. Salomone, J. Schechter and T. Tudron, Phys. Rev. D 23, 1143 (1981); H. Gomm and J. Schechter, Phys. Lett. B 158, 449 (1985); A. A. Migdal and M. A. Shifman, Phys. Lett. B 114, 445 (1982).

11. F. Giacosa, G. Pagliara, Phys. Rev. C76 (2007) 065204; F. Giacosa, arXiv:1110.5923 [nucl-th].

12. D. Parganlija, F. Giacosa and D. H. Rischke, Acta Phys. Polon. Supp. 3 (2010) 963; D. Parganlija, F. Giacosa and D. H. Rischke, arXiv:0911.3996 [nucl-th]; D. Parganlija, F. Giacosa and D. H. Rischke, PoS CONFINEMENT 8 (2008) 070; D. Parganlija, F. Giacosa and D. H. Rischke, AIP Conf. Proc. 1030 (2008) 160.

13. Y. Chen et al., Phys. Rev. D 73 (2006) 014516.

14. D. Parganlija, F. Giacosa, P. Kovacs, G. Wolf, AIP Conf. Proc. 1343 (2011) 328-330; D. Parganlija, F. Giacosa, D. H. Rischke, P. Kovacs, G. Wolf, Int. J. Mod. Phys. A26 (2011) 607-609; D. Parganlija, Acta Phys. Polon. Supp. 4 (2011) 727-732.

15. S. Gallas, F. Giacosa, D. H. Rischke, Phys. Rev. D82 (2010) 014004; S. Gallas, F. Giacosa, D. H. Rischke, PoS CONFINEMENT8 (2008) 089. [arXiv:0901.4043 [hep-ph]]; S. Wilms, F. Giacosa, D. H. Rischke, Int. J. Mod. Phys. E16 (2007) 2388-2393.

16. S. Gallas, F. Giacosa, G. Pagliara, Nucl. Phys. A872 (2011) 13-24.

17. A. Heinz, S. Struber, F. Giacosa and D. H. Rischke, Phys. Rev. D 79 (2009) 037502. A. Heinz, F. Giacosa, D. H. Rischke, [arXiv:1110.1528 [hep-ph]].

18. F. Giacosa, Phys. Rev. D 75 (2007) 054007.

19. R. L. Jaffe, Phys. Rev. D 15, 267 (1977); R. L. Jaffe, Phys. Rev. D 15, 281 (1977); L. Maiani, F. Piccinini, A. D. Polosa and V. Riquer, Phys. Rev. Lett. 93 (2004) 212002 ; F. Giacosa, Phys. Rev. D 74 (2006) 014028; A. H. Fariborz, R. Jora and J. Schechter, Phys. Rev. D 72 (2005) 034001; A. H. Fariborz, Int. J. Mod. Phys. A 19 (2004) 2095; M. Napsuciale and S. Rodriguez, Phys. Rev. D 70 (2004) 094043; F. Giacosa, G. Pagliara, Nucl. Phys. A833 (2010) 138-155. 